\title{
A Forgetful Experience: A Case of Transient Global Amnesia
}

Kalpa Shah MD, PGY2, Chris Sheasgreen MD, PGY 3, Ameen Patel, MB

\section{About the Authors}

Kalpa Shah is a postgraduate PGY3 resident in Internal Medicine, Christopher Sheasgreen is a PGY4 resident in his Gastroenterology fellowship, and Ameen Patel is a staff member of the Division of General Internal Medicine and holds the William J. Walsh Chair in Medical Education, all at McMaster University, in Hamilton, Ontario. Correspondence may be directed to Ameen Patel patela@mcmaster.ca

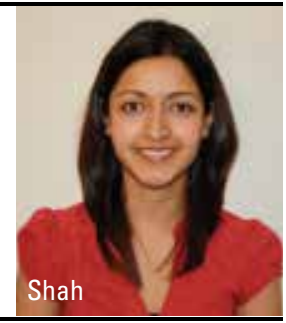

\begin{abstract}
Summary
We present a case of a 67-year-old man with transient global amnesia, a clinical syndrome that presents with an acute onset of temporary amnesia lasting less than 24 hours, without impairment of consciousness or cognition. In this article, we discuss the clinical criteria for diagnosis, the pathophysiology of the condition, and an approach to its diagnostic work-up and prognosis. It is important to differentiate TGA from other entities because it is a benign condition that does not require treatment.
\end{abstract}

\section{Résumé}

Nous présentons le cas d'un homme de 67 ans en ictus amnésique, syndrome clinique caractérisé par un trouble mnésique transitoire d'installation subite qui dure moins de 24 heures, sans altération de la conscience ou de la cognition. Nous examinons les critères cliniques du diagnostic, la physiopathologie du trouble, une méthode d'investigation diagnostique et le pronostic. Il est important de distinguer l'ictus amnésique d'autres troubles étant donné que ce syndrome bénin ne nécessite pas de traitement.

$\mathrm{T}$ ransient global amnesia (TGA) is a clinical syndrome that presents with an acute onset of temporary amnesia lasting less than 24 hours, without impairment of consciousness or cognition. It has an incidence of 3 to 8 per 100,000 people per year. ${ }^{1}$ Approximately $75 \%$ of TGA episodes occur in individuals between 50 and 70 years of age. ${ }^{2}$ It is uncommon in patients younger than 40 years of age. Although various pathophysiologic explanations have been put forth, the etiology of TGA remains uncertain.

\section{Case Presentation}

A 67-year-old man presented to the emergency department with confusion and amnesia. Earlier that day, he had awoken feeling completely normal. He met some friends for coffee at a mall, as per his usual routine. His friends reported no unusual behaviour or confusion. The last thing he remembered is having bid goodbye to his friend at 12:30 p.m., and he had no recollection of events after having left his friend. 
His son had found him at home approximately two hours later, in his underclothes, repeatedly opening and closing the automated garage door and asking whether his son knew where the car was. His son reports having found his father's clothes strewn across the main floor of their home. The patient did not recollect that his wife had taken the car after dropping him off at the mall. During the episode he was alert and responsive. He was oriented to person but disoriented to place. He was subsequently brought to the emergency department.

He had no recent travel history, sick contacts, or infectious symptoms. He had no bowel or bladder incontinence, no smell or taste sensations, and no visual or visceral (epigastric rising) auras. There were no complaints of focal weakness, numbness, tingling, slurred speech, vertigo, diplopia, chest pain, shortness of breath, nausea, vomiting, diarrhea, or fevers and chills. There was no history of prior similar episodes.

He had no medical conditions, was in excellent general health, and did not take any prescription or over-the-counter medications. There was no history of tobacco or recreational drug use, and he used alcohol rarely. He had no family history of neurologic or psychiatric disease.

On examination he had a heart rate of 82 beats per minute and a blood pressure of 152/97 $\mathrm{mm} \mathrm{Hg}$. He was afebrile and had an oxygen saturation of $96 \%$ on room air. He was oriented to person, place, and time. The examination of his head and neck, heart, lungs, abdomen, and extremities were normal. A neurologic examination revealed anterograde amnesia from approximately 12:30 p.m. Long-term memory was preserved. There was no evidence of hallucinations or delusions, and he was able to correctly identify his family members. He had no focal motor, sensory, coordination, or cranial nerve deficits. His reflexes were $2+$ in the upper and lower extremities. Plantar reflexes were down going bilaterally. His speech was fluent and without evidence of aphasia or dysarthria. During the examination, he repeatedly asked what had happened to him. Laboratory investigations showed a normal complete blood count, urea, creatinine, electrolytes, glucose. A liver panel consisting of total bilirubin, alanine transaminase (ALT), alkaline phosphatase, and international normalized ratio (INR) were within normal ranges.

Venous blood gas analysis showed a pH of 7.35 (7.32-7.42) and a partial carbon dioxide pressure ( $\left.\mathrm{PcO}_{2}\right)$ of $52(38-50) \mathrm{mm}$ $\mathrm{Hg}$. Additional blood work revealed a thyroid-stimulating hormone (TSH) level of $1.2(0.3-4.2) \mathrm{mIU} / \mathrm{L}$ and a C-reactive protein (CRP) level of 2.7. The result of a urine toxicology screen was negative, and serum levels of acetaminophen and salicylate were undetectable. Electrocardiography revealed normal sinus rhythm. The results of chest radiography, brain computed tomography $(\mathrm{CT})$, magnetic resonance imaging, and electroencephalography were normal.

His amnesia resolved over 24 hours, and he had no ongoing symptoms of anterograde amnesia. There have been no recurrences in the six months since his discharge.

\section{Discussion}

The term "transient global amnesia" was originally used by Fisher and Adams in 1964 to describe a clinical syndrome characterized by the abrupt onset of anterograde amnesia accompanied by repetitive questioning lasting minutes to hours. ${ }^{3}$ With the exception of amnesia, there were no other neurologic deficits, and patients were alert, responsive, and aware of their personal identity during the attacks. Since then, diagnostic criteria for TGA have been developed by Caplan and later validated by Hodges and Warlow in a prospective study. ${ }^{4}$ Hodges and Warlow followed 153 patients who met the diagnostic criteria for TGA (Table 1) and found that the rate of vascular events (a vascular event being defined in the study as myocardial infarction, stroke, or death) in the TGA group was not increased compared to the general population at 6 months after the episode of TGA. These findings are in keeping with those of a multicentre study in Denmark and prospective follow-up studies of TGA patients, published by the Mayo Clinic. ${ }^{5,6}$ For a diagnosis of TGA to be made, all criteria listed in Table 1 must be met. If only some of the criteria are met - particularly if there are neurologic deficits -the likelihood of alternative diagnoses is greater.

\section{Table 1.}

\section{Clinical Criteria for the Diagnosis of}

Transient Global Amnesia

- Presence of an attack of anterograde amnesia (most of the attack should be witnessed)

- No clouding of consciousness or loss of personal identity

- Cognitive impairment limited to amnesia (no apraxia, aphasia, etc.)

- No focal neurologic or epileptic signs during or after the attack - No recent history of head trauma or seizures

- Resolution of symptoms within 24 hours

Source: Adapted from Hodges JR, Warlow CP.4

\section{Etiology}

The clinical symptoms of difficulty in the formation and retrieval of memories in TGA implicate the hippocampus and the temporal lobe as potential areas of involvement. Several pathophysiological explanations have been proposed, such as migraine-related mechanisms, ischemia, and epileptic phenomena, as well as venous flow abnormalities. ${ }^{7}$ Different modalities of neuroimaging studies have been used to investigate the area of the brain and mechanism involved in TGA. everal studies using single-photon emission CT show a decrease in 
cerebral blood flow in the temporal lobe and hippocampal region in most patients. Studies with diffusion-weighted imaging (DWI) have yielded conflicting results, some studies showing no abnormalities on DWI and other studies describing small lesions in the hippocampal region. ${ }^{8}$ The etiology of TGA continues to be shrouded in controversy.

\section{Differential Diagnosis}

The differential diagnosis of TGA includes complex partial seizure, transient ischemic attack, acute confusional state, transient epileptic amnesia, dissociative amnesia, intoxication, and Wernicke-Korsakoff syndrome.

It is often difficult to differentiate TGA from transient ischemic attack (TIA); some data suggest that TGA is a result of an ischemic process involving the hippocampus. ${ }^{8}$ Findings of focal neurologic deficits, such as motor weakness or sensory abnormalities, favour the diagnosis of TIA. In addition, the presence of risk factors such as smoking, hypertension, and dyslipidemia should increase the clinical suspicion for TIA.

In patients with TGA, self-identity is preserved, which differentiates TGA from some psychiatric disorders such as dissociative amnesia, in which personal identity is lost. Furthermore, attention is intact in episodes of TGA, in contrast to drug-related confusional states in which patients may present with attention deficits.

Patients who are experiencing complex partial seizures often display automatisms, staring spells, and impaired consciousness; whereas in TGA, patients are alert. It is more challenging to differentiate TGA from a complex partial seizure when patients present in a postictal state.

Patients with Wernicke-Korsakoff syndrome will have a history of heavy alcohol use, and the clinical presentation will be characterized by anterograde as well as retrograde amnesia and confabulation. There may also be other clues on examination and laboratory data to support excessive alcohol use.

\section{Work-Up}

It is important to note that TGA is a clinical diagnosis and remains a diagnosis of exclusion. The diagnostic criteria as outlined by Hodges and Warlow act as a tool to help guide clinicians. If all the criteria are met, the probability of a diagnosis of TGA is increased. An initial work-up should include serum electrolytes, glucose, oxygenation status, and a toxicology screen. It is often useful to perform brain imaging to rule out other serious causes such as trauma and stroke. In the absence of recurrent episodes, risk factors for seizures (such as a prior stroke), or a history suggestive of epileptic activity, it is not necessary to perform electroencephalography.

\section{Treatment and Prognosis}

A benign condition with no long-term sequelae, TGA requires no treatment. The reported annual rate of recurrence varies between studies but is within the range of $5 \%$ to $8 \% .{ }^{2,9}$ There is no published literature that indicates a subsequent risk of delirium or neurodegenerative diseases in patients who have experienced TGA.

\section{Conclusion}

Transient global amnesia (TGA) is a syndrome of reversible amnesia of unclear etiology. It occurs most frequently in individuals between 50 and 70 years of age. It is important to differentiate TGA from other causes of neurologic impairment, as it is a benign condition that does not require treatment. Applying the diagnostic criteria can help clinicians determine the probability of a diagnosis of TGA. For cases that do not meet all of the diagnostic criteria, further investigations should be considered to rule out more serious conditions that require intervention. Diseases may not necessarily present as described in the literature and oftentimes will help unveil the diagnosis.

\section{References}

1. Miller JW, Petersen RC, Metter EJ, et al. Transient global amnesia: clinical characteristics and prognosis. Neurology 1987;37:733-7.

2. Quinette P, Guillery-Girard B, Dayan J, et al. What does transient global amnesia really mean? Review of the literature and thorough study of 142 cases. Brain 2006;129:1640-58.

3. Fisher CM, Adams RD. Transient global amnesia. Trans Am Neurol Assoc 1958;83:143-6.

4. Hodges JR, Warlow CP. Syndromes of transient amnesia: towards a classification. J Neurol Neurosurg Psychiatry 1990;53:834-43.

5. Miller JW, Petersen RC, Metter EJ, et al. Transient global amnesia: clinical characteristics and prognosis. Neurology 1987;37:733-7.

6. Hinge $\mathrm{H}$, Jensen TS, Kjaer M, et al. The prognosis of transient global amnesia: results of a multicentre study. Arch Neurol 1986;43:673-6.

7. Bartsch T, Deuschl G. Transient global amnesia: functional anatomy and clinical implications. Lancet Neurol 2010;9:205-14.

8. Sander K, Sander D. New insights into transient global amnesia: recent imaging and clinical findings. Lancet Neurol 2005;4:437-44.

9. Pantoni L, Bertini E, Lamassa M, et al. Clinical features, risk factors and prognosis in transient global amnesia: a follow-up study. Eur J Neurol 2005; 12:350-6. 